Revue internationale P.M.E.

Économie et gestion de la petite et moyenne entreprise

Revure

internationale

PME

\title{
Les facteurs de diffusion et de pénétration des nouvelles technologies dans les PME québécoises
}

\section{Pierre-André Julien, Jean-Bernard Carrieres et Louis Hébert}

Volume 1, numéro 2, 1988

URI : https://id.erudit.org/iderudit/1007881ar

DOI : https://doi.org/10.7202/1007881ar

Aller au sommaire du numéro

Éditeur(s)

Presses de l’Université du Québec

ISSN

0776-5436 (imprimé)

1918-9699 (numérique)

Découvrir la revue

Citer cet article

Julien, P.-A., Carrieres, J.-B. \& Hébert, L. (1988). Les facteurs de diffusion et de pénétration des nouvelles technologies dans les PME québécoises. Revue internationale P.M.E., 1(2), 193-222. https://doi.org/10.7202/1007881ar
Résumé de l'article

Cet article traite des facteurs ou variables internes à la firme pouvant influencer la modernisation du processus de production des PME, particulièrement dans trois sous-branches industrielles soit les industries des produits de plastique, des ateliers d'usinage et des scieries.Les résultats de l'analyse montrent que dans le cas des firmes recourant aux technologies à contrôle numérique, il s'agit, en général, d'entreprises un peu plus grandes que la moyenne avec, à leur tête, un propriétaire-dirigeant plus scolarisé et même diplômé universitaire et de formation technique. Ces entreprises possèdent une gestion relativement "complexe», dont les sources d'approvisionnement sont géographiquement plus diversifiées et dont les exportations sont relativement importantes. Mais surtout, ces entreprises recourent à des sources d'informations technologiques très spécialisées telles que fournisseurs, foires industrielles, littérature technique spécialisée et centres de recherche privés ou publics. 


\title{
Les facteurs de diffusion et de pénétration des nouvelles technologies dans les PME québécoises*
}

\author{
Pierre-André JULIEN \\ Jean-Bernard CARRIERES \\ Louis HÉBERT \\ GREPME, Université du Québec à Trois-Rivières
}

\begin{abstract}
RÉSUMÉ
Cetarticle traite des facteurs ou variables internes à la firme pouvant influencer la modernisation du processus de production des PME, particulièrement dans trois sous-branches industrielles soit les industries des produits de plastique, des ateliers d'usinage et des scieries.

Les résultats de l'analyse montrent que dans le cas des firmes recourant aux technologies à contrôle numérique, il s'agit, en général, d'entreprises un peu plus grandes que la moyenne avec, à leur tête, un propriétaire-dirigeant plus scolarisé et même diplômé universitaire et de formation technique. Ces entreprises possèdent une gestion relativement acomplexem, dont les sources d'approvisionnement sont géographiquement plus diversifiées et dont les exportations sont relativement importantes. Mais surtout, ces entreprises recourent à des sources d'informations technologiques très spécialisées telles que foumisseurs, foires industrielles, littérature technique spécialisée et centres de recherche privés ou publics.
\end{abstract}

\section{ABSTRACT}

In this paper, we study the impact of different internal variables on the modernization of the production process in small manufacturing business, particularly in plastic, machine tools and sawmill industries.

The results show that the modern firm is greater than the other, has a leader more scolarized and even with university diploma and technical formation, which importation and exportation are more important. But before all, the first variable is the good using of technological information, more specialized, coming from producers from industrial fairs, from technical journals and from public or private research centers.

\section{RESUMEN}

Este articulo trata de los factores o variables internos que pueden influenciar la modernizacion del proceso de produccion en las pequenas y medianas empresas, particularmente en tres sectores industriales, a saber, las industrias de productos plasticos, los talleres mecanicos de fabricacion ajuste o rectificacion y las serrerias.

Los résultados revelan que en el caso de las firmas que recurren a tecnologias de control numérico, se trata por lo general de empresas un poco mas grandes que la media, que tienen a la cabeza un propietario-dirigente mas instruido, incluso diplomade universitario con formacion técnica, y que ejerce una gestion relativamente compleja, cuyas fuentes de aprovisionamiento estan geograficàmente mas diversificadas y cuyas importaciones son relativamente importantes. Pero, sobretodo, estas empresas recurren a fuentes de informacion tecnologicas bastante especializadas, tales como los abatecedores, las ferias industriales, las publicaciones téenicas especializadas y los centros de investigacion privados y publicos.

* Cet article repose sur une recherche qui a été rendue possible grâce à une subvention du Conseil de la Science et de la Technologie du Québec. 


\section{Introduction}

Le besoin de modernisation du processus de production des PME dans le cadre de la présente révolution technologique n'est plus à prouver. Déjà les entreprises multinationales réorientent leur stratégie en augmentant leur production dans les pays à faibles salaires et en investissant massivement dans les nouvelles technologies de production. Leurs moyens financiers et les aides de l'État leur permettent d'agir ainsi. Mais il n'en est pas de même du côté de la PME, même si un grand nombre d'entre elles, non protégées par des créneaux de long terme, subissent les mêmes contraintes de coûts et de concurrence.

Mais l'introduction de nouvelles technologies dans les entreprises, en particulier dans les PME, est un problème complexe. Si leur modernisation semble nécessaire voire impérieuse pour adapter les structures industrielles face à la concurrence nationale et internationale, quantité de facteurs peuvent influencer négativement ou positivement le mouvement de substitution technologique. Il semble même que beaucoup de PME tardent à emprunter le virage technologique et se laissent ainsi dangereusement distancer par les grandes entreprises. Or, compte tenu de l'importance des PME dans l'économie, de leur capacité particulière à créer des emplois et de leur impact régional, il est primordial qu'elles empruntent le virage technologique pour faire face aux nouveaux défis de l'économie mondiale.

Il est vrai que de nombreux facteurs peuvent faciliter le passage des PME vers l'usine du futur. Ainsi, les producteurs des équipements tendent de plus en plus à orienter leurs produits vers les besoins et les possibilités de celles-ci. De plus, leur faible taille et leur flexibilité si souvent constatées les dégagent des problèmes de conversions coûteuses. Enfin les PME se montrent souvent plus innovatrices que les grandes entreprises, augmentant d'autant leurs chances de s'adapter rapidement aux nouvelles technologies. Cependant, d'autres études montrent que plusieurs obstacles subsistent. Ils peuvent provenir du manque d'adéquation des nouveaux équipements pour les PME ou de leur coût trop élevé, compte tenu d'une capacité financière limitée ou d'un haut niveau d'endettement. Ils peuventégalement venir du propriétairedirigeant qui manque souvent d'information sur les caractéristiques et les possibilités des nouvelles technologies. Parallèlement, la PME ne possède pas toujours, au sein de son personnel, l'expertise nécessaire pour opérer une machinerie complexe. D'autres résistances coûteuses peuvent provenir du personnel de l'entreprise qui, lui aussi faute d'informations, comprend mal les motifs de modernisation et craint de voir disparaître ses emplois. 


\section{Les fondements théoriques de la recherche}

Il y a encore peu de temps, aucune recherche s'adressant aux PME plutôt qu'aux grandes entreprises ne s'était penchée sur ce problème. Par chance, le récent regain d'intérêt pour les petites entreprises a permis la production de nouvellesétudes sur l'innovation technologique et les PME. Dans notre recherche, nous avons voulu appliquer la théorie micro-économique de la firme, portant sur la diffusion technologique et analysée particulièrementaux États-Unis, auxPME manufacturières québécoises de façon à mesurer l'importance relative des facteurs surtout internes expliquant cette diffusion dans les firmes. Pour ce faire, nous avons négligé, il est vrai, les variables macro-économiques et historiques qui auraient permis une compréhension plus globale du phénomène comme l'expliquent bien Bouchut et Jacot (1986) ou Pastré (1984). Les PME étudiées évoluaient dans trois branches industrielles peu concentrées, soit des industries de produits de plastique, des ateliers d'usinage et des scieries. Ces trois secteurs nous semblaient représentatifs de l'économie québécoise dans sa partie plus turbulente (les produits de plastique et les nouvelles matières plastiques), dans ses firmes évoluant dans l'ombre des grandes entreprises (les ateliers d'usinage), ou encore dans ses entreprises plus traditionnelles (les scieries).

Dans la théorie de la firme de la diffusion de la technologie, Edwin Mansfield est sans contredit un pionnier. Ses nombreuses études se sont concentrées sur l'explication du délai d'adoption d'une innovation ou encore sur la rapidité avec laquelle l'usage d'une innovation technologique se répand dans une industrie. Il a d'abord mis en évidence le rôle majeur de la «profitabilité» de l'innovation et de la taille de l'investissement nécessaire. Mansfield a aussi testé d'autres facteurs comme le taux de croissance de la firme, sa rentabilité, son évolution récente, les liquidités disponibles ainsi que la personnalité et l'âge de son président. De plus, il a soutenu que le manque de connaissances sur l'innovation et les résistances aux changements de la part des dirigeants pouvaient s'avérer des obstacles majeurs à cette diffusion (Mansfield et alii, 1971).

Gold et alii (1970) ont plus tard étudié 14 innovations dans la sidérurgie américaine. Ils ont aussi noté que les explications à l'implantation d'une innovation variaient selon que cette innovation venait s'ajouter, déplacer ou remplacer des équipements existants. La profitabilité de l'innovation estencore unélément important mais les auteurs suggèrent que l'adaptabilité de l'innovation aux installations en place et que les "coûts d'opportunité» et de conversion entraînés sont aussi à considérer. D'autres éléments, comme par exemple la concurrence au sein d'une industrie, influenceraient positivement la vitesse de diffusion. De même, une innovation se diffuse beaucoup plus rapidement dans une industrie connaissant une forte croissance.

Mais ces éléments surtout économiques et techniques doivent être complétés par des facteurs sociologiques ou organisationnels. Carter et Williams (1957 et 
1959), dans une étude très fouillée, avaient déjà montré l'importance du profil du management sur la propension d'une firme à adopter les dernières technologies. Des critiques de certains aspects méthodologiques de cette recherche ont cependant nui aux résultats trouvés. C'est ce qui explique que d'autres auteurs ont repris divers éléments del'étude. Par exemple, Cohn (1980) a démontré que la présence d'ingénieurs et de diplômés dans la firme, l'utilisation de consultants et la tenue de réunions interdépartementales pour les cadres semblaient distinguer clairement les entreprises utilisant des technologies avancées des autres firmes. Soulignons que ces facteurs se rapportent avant tout aux ressources humaines, comme l'avaient dit Carter et Williams. L'ouvrage de Rogers et Shoemaker (1971) va encore plus loin et identifie un profil général des individus considérés comme innovateurs ou retardataires. Selon eux, les innovateurs seraient dans l'ensemble des individus jeunes, scolarisés, de classe sociale élevée, moins dogmatiques, qui ont des attitudes favorables relativement à tout ce qui concerne le changement, le risque, la science, et qui ont un besoin élevé d'accomplissement, ce qui est discutable. Mais surtout, ils ont mis en évidence le rôle que peuvent avoir certaines personnes dans le processus de diffusion, soit des gens disposant d'informations sur les innovations («technology gatekeepen ou «sentinelles») ou qui se font les promoteurs d'une innovation (les «champions»). Il peut également s'agir de personnes dont le leadership, le statut, la respectabilité, le pouvoir et les valeurs peuvent influencer la décision d'autres gens ou d'organisations. De même, l'accès à des sources nombreuses et variées d'informations ainsi que la possibilité de disposer très tôt d'informations sur l'innovation semblent être des variables importantes.

Duchesneau et alii (1979) ainsi qu'à nouveau Cohn et Turyn (1980 et 1984) ont pour leur part examiné les relations entre la complexité, la centralisation, la formalisation et l'utilisation de certains équipements industriels avancés. Le nombre d'innovations technologiques implantées variait directement avec la complexité de l'organisation mais inversement avec sa centralisation et sa formalisation. Ils ont remarqué que les entreprises les plus innovatrices, c'est-à-dire celles qui avaient implanté le plus grand nombre d'innovations faisaient partie des organisations les plus «complexes». De plus, ces entreprises possédaient un personnel cadre plus scolarisé et plus spécialisé.

L'exposition à un grand nombre de sources d'informations influencerait aussi la propension d'une entreprise manufacturière à innover. Dans son étude sur l'industrie du textile, Olsen (1974) a montré que le grand nombre de fournisseurs d'équipement et l'utilisation de la littérature technique étaient directement correlés à l'implantation d'innovations. De plus, comme le suggéraient Rogers et Shoemaker, la présence de «sentinelles techniques» et de «champions» qui supportaient les projets d'innovations a été, selon lui, une variable déterminante.

Cette analyse d'un ensemble de recherches, de publications, etc. permet donc de faire ressortir les facteurs importants ou les variables déterminantes (significatives) de l'adoption d'une innovation technologique en milieu industriel. 
Ces facteurs, nous les avons regroupé en trois catégories, soit :

1. les caractéristiques de l'innovation

- la «profitabilité» et le coût

2. les caractéristiques de l'industrie

- le taux de croissance

- la concurrence

3. les caractéristiques de la firme

a) caractéristiques structurelles

- la taille

- la croissance

- la disponibilité d'information

b) le profil du management

- l'âge, la scolarité, la formation des cadres et du propriétairedirigeant

- la compétence technique de la firme

c) les caractéristiques organisationnelles

- la «complexité»

- la présence de «sentinelles technologiques» et de «champions»

- la communication et la coordination efficaces

Le groupe de caractéristiques de l'innovation se divise en deux. D'abord, les caractéristiques primaires de l'innovation seraient celles qui sont indépendantes ou plutôt qui ne dépendent pas des perceptions et de l'attitude des utilisateurs. La «profitabilité» et le coût de l'innovation constitueraient ce type de caractéristiques. Ils affecteraient respectivement de manière positive et négative la vitesse et le degré de diffusion. Dans la notion de coût, l'on inclut autant le coût direct d'acquisition que les coûts de conversion, de rodage et d'opportunité. Ensuite les caractéristiques secondaires sont celles qui sont influencées par les perceptions des utilisateurs potentiels et dont la valeur varie par conséquent selon les organisations. On retrouve sous ce vocable les attributs d'une innovation définis par Rogers et Shoemaker (1971 et 1975). Le sens ou la valeur de ces attributs peuvent en effet varier selon l'organisation qui considère l'innovation. L'une pourra la juger complexe et l'autre très complexe sans que l'on puisse en connaître la complexité véritable (absolue).

Le deuxième groupe de facteurs résiderait dans les caractéristiques de l'environnement de la firme. Néanmoins, c'est surtout l'environnement sectoriel de la firme, sa branche industrielle qui nous intéresse particulièrement ici. Dans cette perspective, la connaissance interne de l'industrie et son taux de croissance seraient d'importants facteurs d'accélération de la diffusion. 
Le troisième groupe serait constitué des caractéristiques générales, managériales et organisationnelles de l'entreprise. Outre le niveau de croissance et la disponibilité d'informations, la taille de la firme est considérée comme une variable importante. Néanmoins, comme nous l'avons fait remarquer précédemment, son influence tantôt positive, tantôt négative sur la diffusion nous empêche de poser un jugement clair et définitif. Plusieurs recherches ont validé, vérifié et confirmé l'hypothèse schumpeterienne quant au rôle de cette variable. En ce sens, la grande entreprise, que ce soit à cause de son potentiel financier (Malecki, 1975) ou de ses efforts supérieurs en R \& D (Rosenberg, 1974), s'avérerait plus innovatrice ou utiliserait plus rapidement une innovation technologique. Cependant, un nombre presque aussi important de recherches sont venues contredire cette hypothèse ou encore en diminuer l'importance. Plusieurs (Gebhart et Hartzold, 1974; Smith, 1974; Hambert, 1963; Kennedy et Thirwall, 1972; Wiches, 1979) ont mis en doute les avantages de la grande dimension et invoqué par exemple la mentalité de «statu quo» et l'inertie des grandes organisations. D'autres (Rothwell et Zegveld, 1982, et OCDE, 1982) ont démontré que les petites et moyennes entreprises concentraient une proportion importante des innovations dans certains secteurs et que cette proportion était souvent supérieure à leur part de marché. La relation entre la taille et la diffusion peut aussi se voir d'une manière dynamique. A cet effet, la taille des firmes les plus innovatrices varierait selon le stade de développement ou la phase du cycle de vie de l'industrie où se produit l'innovation (Kaplinsky, 1983). Ajoutons enfin que le problème qui nous concerne ici n'est pas la création d'une nouvelle technologie mais plutôt son adoption. Dans ce cas, le peu d'études sur le sujet ne permet pas de discriminer entre les petites et les grandes entreprises.

Enfin, les demiers facteurs toucheraient l'organisation de l'entreprise, notamment sa complexité, sa centralisation et sa formalisation ainsi que son ouverture à la communication. Il faut aussi mettre en valeur le rôle joué par certaines personnes, à titre de «sentinelles technologiques» de "champions» ou encore «d'agents de changement» et de «leaders d'opinion». L'attitude et les valeurs de la direction des PME de la firme exerceraient également une grande influence sur les sentiments, l'opinion et les comportements du personnel et de la firme en matière d'innovation.

Toutefois, la plupart du temps, les résultats des recherches ne proviennent de la prise en compte que d'un petit nombre de facteurs. Cette situation se retrouve particulièrement dans l'analyse des caractéristiques de l'entreprise. Peu de recherches ont tracé un portrait plus élaboré ou complet des firmes étudiées. On s'en est tenu habituellement à quelques variables dont le choix repose sur l'orientation professionnelle etscientifique des auteurs. Les recherches qui ont touché lacomplexité, la centralisation et la formalisation sans s'attarder à d'autres variables organisationnelles en sont un exemple.

Laprésente recherchea essayé justement d'intégrer ces différentes dimensions économiques, techniques et managériales qui permettent l'adoption des nouvelles technologies dans les PME manufacturières. Dans cette perspective, elle poursuivait trois buts spécifiques, soit : 
a) d'abord appliquer le corpus théorique de la diffusion d'une innovation technologique en milieu industriel, et plus précisément le cadre d'analyse développé par Carter et Williams (1957) et Cohn (1980), à la PME manufacturière;

b) identifier, étudier et hiérarchiser certaines caractéristiques organisationnelles et managériales qui différencient les PME manufacturières utilisatrices d'une innovation technologique, (le contrôle numérique dans ce cas), des PME non-utilisatrices dans les trois industries;

c) proposer un certain profil des PME utilisatrices.

Pour ce faire nous avons effectué une enquête auprès d'un échantillon d'entreprises des trois industries retenues à partir des facteurs soulevés par cette analyse de la littérature. Ces facteurs constituent la grille d'analyse qui nous a permis de construire le questionnaire d'enquête.

\section{$3 \quad$ Méthodologie}

Concrètement, cette enquête avait pour but de comparer les firmes utilisant des technologies de pointe à celles ne les utilisant pas de façon à faire ressortir les caractéristiques permettant de bien les distinguer. Ces caractéristiques étaient basées sur les facteurs discutés plus haut.

Nous avons divisé ces facteurs en deux catégories de variables, soit une variable dépendante et 4 groupes de variables indépendantes. L'annexe 1, (p. 214218), résume les variables en détail en spécifiant les types de mesure utilisée. Il faut retenir que la variable dépendante que nous avons retenue est dichotomique. Elle prend la valeur «1» lorsqu'une entreprise possède au moins un équipement de production doté du contrôle numérique par ordinateur (C.N.O.) au niveau de n'importe lequel de ses segments de production. Sa valeur est nulle lorsque ce n'est pas le cas.

Les variables indépendantes ont été divisées en quatre groupes, soit celles touchant le profil du propriétaire-dirigeant, le profil des cadres, les caractéristiques générales et structurelles de la firme, et enfin les caractéristiques organisationnelles. En effet, les autres caractéristiques économiques (concurrence internationale, etc.) et technologiques (disponibilité des technologies) sont censées être relativement les mêmes pour toutes les firmes d'une même industrie. Ellesn'ont donc pas été retenues dans le questionnaire sauf dans la description de certaines technologies pour évaluation par le dirigeant d'entreprise.

Ces facteurs nous ont permis de construire deux questionnaires, à questions en bonne partie fermées. Le principal s'adressait au propriétaire-dirigeant de l'entreprise au cours d'entrevues structurées d'environ quatre-vingt-dix minutes effectuées par les chercheurs et les assistants de recherche. Ce premier questionnaire 
comprenait environ 225 questions, dépendant de la complexité des technologies selon les trois industries. Pour y répondre, le propriétaire-dirigeant pouvait au besoin être aidé par un cadre, en particulier pour les questions financières ou de production.

Le second questionnaire, beaucoup plus succint, a été distribué aux cadres de l'entreprise lors de la visite des enquêteurs. Le propriétaire-dirigeant devait retourner le questionnaire dûment rempli par le courrier. En cas de retard, une lettre de rappel lui était adressée. Malheureusement, peu de ce deuxième questionnaire ont été retournés, malgré nos appels répétés. Les résultats à ce propos sont donc plus sujets à caution.

Ces deux questionnaires ont subi un pré-test dans cinq entreprises dont deux de l'industrie des produits de plastique, deux de celle des ateliers d'usinage et une des scieries. Les visites d'entreprises se sont déroulées en février, mars, avril et juin 1987.

Ces questionnaires ont été appliqués à un échantillon d'entreprises des trois industries dont la population comprenait en 1983 près de mille firmes, et dont $98 \%$ avait moins de 200 employés. Nous avons d'abord retenu un premier échantillon probabilistique de 196 entreprises réparties également entre les trois branches industrielles. Quarante-quatre autres ont été identifiées par des experts de l'industrie que nous avons rencontrés comme des firmes avancées au point de vue technologique et ont, par conséquent, été choisies de manière non-probabiliste.

Les deux cent quarante firmes de notre échantillon ont reçu deux lettres, l'une provenant de nous, l'autre de l'organisme commanditaire, pour les inviter à participerà la recherche. En tout, soixante et onze firmes, dont 29 considérées comme modemes, ont répondu affirmativement à cette démarche et ont pu être visitées. Cet échantillon représente ainsi plus de $7 \%$ de la population totale ou environ $4,7 \%$ des entreprises n'utilisant pas le contrôle numérique et $32,2 \%$ de celles l'utilisant. La représentativitéau total est beaucoup plus élevée comparée à la plupart des recherches effectuées dans ce domaine et les résultats peuvent être généralisés aux trois industries retenues en tenant compte des différences entre l'échantillon et lapopulation telles que résumées au tableau 1 (p. 201).

En particulier, les entreprises de notre échantillon, tout en étant à $98 \%$ des PME ayant moins de 200 employés, sont en moyenne plus grandes que pour la population. Cecis'explique parle biais en faveur desfirmes recourant aux équipements à contrôle numérique et à deux scieries ayant plus de 200 employés, qui viennent hausser le pourcentage moyen d'employés. Notre échantillon comprenait toutefois de très petites entreprises (ayant moins de 5 employés). Ce biais vient aussi augmenter la moyenne des ventes de l'échantillon comparée avec celle de la population.

Les autres caractéristiques de l'échantillon sont toutefois représentatives de la population. Ainsi les entreprises de notre échantillon ont en moyenne 20,7 ans d'existence, la plus jeune ayant quatre ans et les plus vieilles, 83 ans. Elles ont de plus à leur tête un propriétaire-dirigeant de 47 ans en moyenne, qui, comme on le verra plus loin (tableau 2) pour $32 \%$ d'entre elles (23 firmes), possède au moins un 
baccalauréat et pour $38 \%$ (27) un diplôme d'études collégiales. Vingt-six d'entre elles ont au moins un ingénieur à leur service et vingt-neuf ont formé un comité de gestion.

De plus, 29 (41\%) de nos entreprises ont un ordinateur. Enfin, le même nombre disposent de la technologie du CNO. Ces équipements sont généralement en place depuis 54,9 mois et représentent un investissement de $345885 \$$ en moyenne.

Tab. 1. Profil statistique des PME de l'échantillon et de la population Industries de produits de plastique, des ateliers d'usinage et des scieries

\begin{tabular}{|c|c|c|c|c|c|c|}
\hline \multirow[t]{2}{*}{ a) } & \multirow[t]{2}{*}{ PROFIL GÉNÉRAL } & \multicolumn{4}{|c|}{ ECHANTILLON } & \multirow[t]{2}{*}{ POPULATION } \\
\hline & & moyenne & ecart type & minimum & maximum & \\
\hline & 20,68 & 1,93 & 4,00 & 83,00 & - \\
\hline \multicolumn{2}{|c|}{$\begin{array}{l}\text { nombre d'emplois } \\
\text { valeur des ventes en } 86\end{array}$} & 47,76 & 9,80 & 2,00 & 594,00 & 27,8 \\
\hline \multicolumn{2}{|c|}{$\left({ }^{1000 \$)}\right.$} & 4509,51 & 1166,03 & 90,00 & 60000,00 & 1703,00 \\
\hline \multirow{2}{*}{\multicolumn{2}{|c|}{$\begin{array}{l}\text { âge du propriétaire-diri- } \\
\text { geant } \\
\text { nombre moyen de cadres par } \\
\text { PME }\end{array}$}} & 47,28 & 1,22 & 25,00 & 65,00 & - \\
\hline & & 4,17 & 0,40 & 1,00 & 17,00 & - \\
\hline \multirow[t]{5}{*}{ c) } & TAILLE DES ENTREPRISES & \multicolumn{2}{|c|}{ ÉCHANTILLON } & \multicolumn{2}{|c|}{ POPULATION } & \\
\hline & & nombre & $\%$ & nombre & $\%$ & \\
\hline & moins de 50 employés & 54 & 76,0 & 823 & 86,0 & \\
\hline & de 50 à 99 employés & 9 & 13,0 & 79 & 8,3 & \\
\hline & plus de 100 employés & 8 & 11,0 & 55 & 5,7 & \\
\hline
\end{tabular}

Sources : Nos calculs; «Forme juridique et taille des établissements manufacturiers au Québec 1983n, Bureau de la statistique du Québec, 1987

\section{$4 \quad$ Les résultats de l'enquête}

Les résultats de l'enquête touchent les caractéristiques des firmes, leurs objectifs, les sources d'information des technologies utilisées et les barrières à l'innovation. Ces facteurs seront mis en relation avec l'adoption de la technologie du contrôle numérique (CN). La très faible diffusion, pour ne pas dire la quasi-absence, des autres technologies encore plus avancées implique ce choix. 


\subsection{Les caractéristiques des firmes}

Nous nous attarderons tout d'abord aux variables qui distinguent les entreprises dotées d'équipements à contrôle numérique (CN) de celles qui ne le sont pas, notamment les variables générales, managérielles et organisationnelles. Ceci nous permettra du même coup de pouvoir identifier certains facteurs explicatifs de l'adoption de la technologie du CN.

\subsubsection{Les caractéristiques managériales générales et organisationnelles}

Comme on peut le voir au tableau 2, (p. 203-205), les entreprises qui utilisent des équipements à contrôle numérique ont, en général, un propriétaire-dirigeant plus scolarisé (21 années d'étude contre 16 années pour les autres PME), disposant 2,5 fois plus souvent d'un diplôme universitaire. Ces dirigeants sont aussi dotés d'une meilleure formation technique; plusieurs sont des ingénieurs. Ces trois premières caractéristiques sont très importantes pour différencier les firmes modernes des autres comme le démontrent les tests statistiques.

Toutefois, il y a peu de différence sur la variable évaluant les attitudes visà-vis des nouvelles technologies. Il est possible que le type de questions retenues ou l'échelle à cinq niveaux ne permettent pas de bien différencier les attitudes.

Pour les caractéristiques structurelles, on peut voir aussi que les firmes utilisatrices d'équipements avec $\mathrm{CN}$ réalisent une plus grande part de leurs achats à l'extérieur du Québec, exportent presque deux fois plus souvent et ont un nombre à peine inférieur de fournisseurs comparativement aux entreprises sans $\mathrm{CN}$.

Surtout, elles collaborent beaucoup plus souvent avec des organismes de recherche et utilisent plus fréquemment des sources d'informations comme la littérature technique, les fournisseurs et les foires industrielles. Ces caractéristiques, sauf la troisième, sont aussi statistiquement très discriminantes.

Les firmes modernes ont en moyenne deux fois plus d'ingénieurs, mais presqu'autant de techniciens et de cadres à la production.

Contrairement à ce que la théorie laisse supposer, ces entreprises qui utilisent le $\mathrm{CN}$ n'ont pas connu une croissance supérieure de leurs ventes depuis cinq ans alors que leur performance, inférieure en ce qui concerne l'emploi, laisse croire (ce qui était à prévoir) que le $\mathrm{CN}$ a un impact négatif à ce niveau comparé aux autres entreprises. Faute de réponses suffisantes, nous n'avons pu comparer l'évolution des actifs.

Comme on l'a dit, les firmes modernes ont beaucoup plus souvent un ordinateur, recourent un peu plus à des consultants et utilisent un peu plus souvent un système de prix de revient, sans que ces variables soient très discriminantes. 
Tab. 2. Résultats : moyenne des firmes utilisatrices et des non-utilisatrices Industries des produits de plastique, des ateliers d'usinage et de scierie

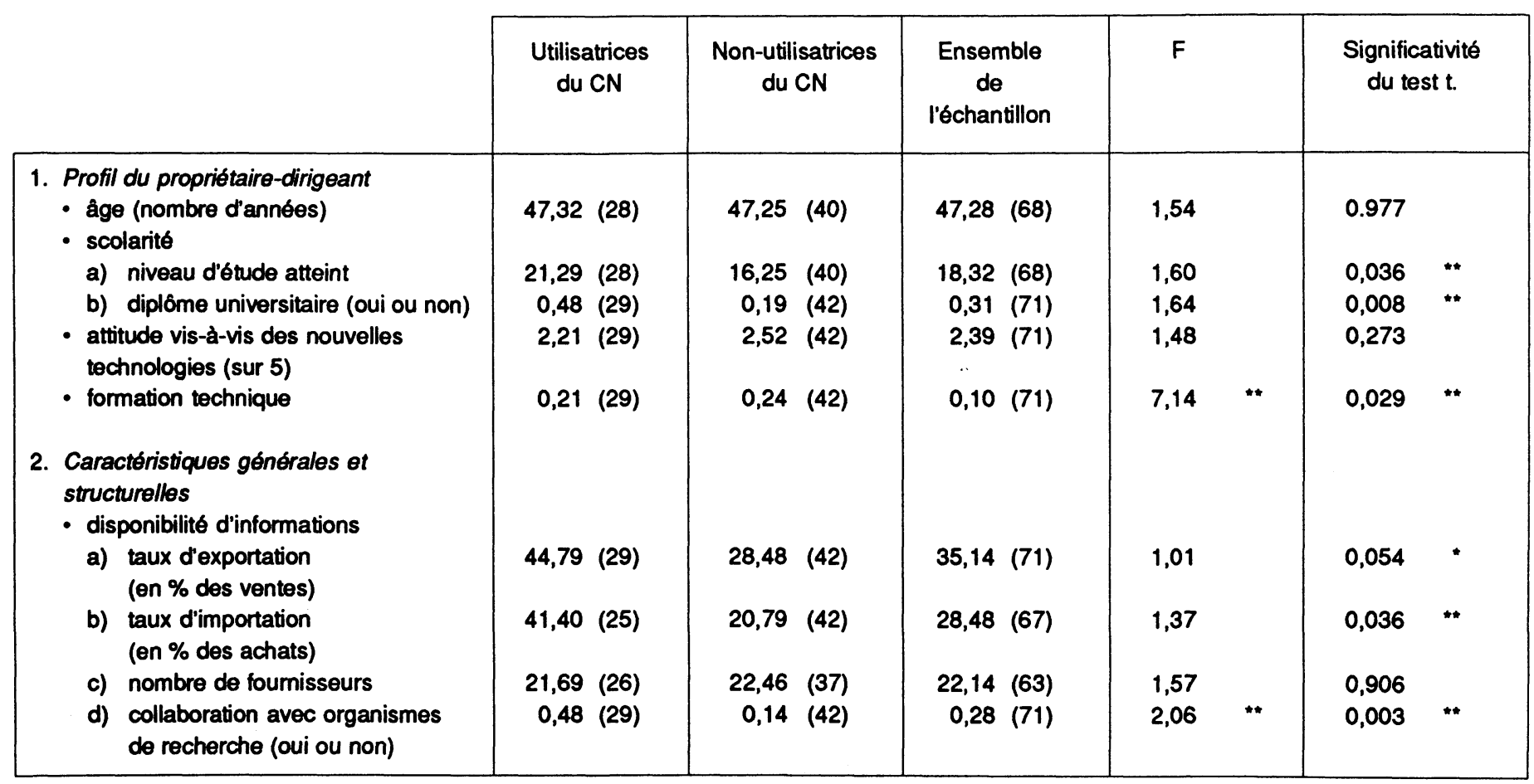

- Différence significative à $90 \%$

** Difference significative à $95 \%$ 
Tab. 2. (suite) Résultats : moyenne des firmes utilisatrices et des non-utilisatrices Industries des produits de plastique, des ateliers d'usinage et de scierie

\begin{tabular}{|c|c|c|c|c|c|c|c|c|}
\hline & $\begin{array}{l}\text { Utilisatrices } \\
\text { du } \mathrm{CN}\end{array}$ & $\begin{array}{c}\text { Non-utilisatrices } \\
\text { du } \mathrm{CN}\end{array}$ & $\begin{array}{c}\begin{array}{c}\text { Ensemble } \\
\text { de } \\
\text { l'échantillon }\end{array} \\
8,39(70)\end{array}$ & \multicolumn{2}{|l|}{$F$} & \multicolumn{2}{|c|}{$\begin{array}{l}\text { Significativité } \\
\text { du test t. }\end{array}$} \\
\hline $\begin{array}{l}\text { e) } \\
\text { - com } \\
\text { a) } \\
\text { b) } \\
\text { c) } \\
\text { croi } \\
\text { ann } \\
\text { a) } \\
\text { b) } \\
\text { c) } \\
\text { prat } \\
\text { a) } \\
\text { b) } \\
\text { c) }\end{array}$ & $\begin{array}{l}\text { fréquence d'utilisation de sources } \\
\text { d'information (littérature technique, } \\
\text { foires industrielles, foumisseurs) } \\
\text { (sur 15) } \\
\text { npétence technique } \\
\text { nombre d'ingénieurs } \\
\text { nombre de techniciens } \\
\text { \% cadres en production } \\
\text { issance (taux de croissance } \\
\text { luel } 81-86 \text { ) } \\
\text { emploi } \\
\text { ventes } \\
\text { actifs } \\
\text { tiques de gestion } \\
\text { usage d'un ordinateur (oui ou non) } \\
\text { utilisation de consultants } \\
\text { (oui ou non) } \\
\text { réalisation d'études de marché } \\
\text { (oui ou non) }\end{array}$ & $\begin{array}{rr}0,81 & (26) \\
3,25 & (28) \\
0,33 & (29) \\
& \\
7,04 & (20) \\
26,58 & (23) \\
- & \\
0,76 & (29) \\
0,52 & (29) \\
& \\
0,45 & (29)\end{array}$ & $\begin{array}{rr}9,31 & (33) \\
21,31 & (29) \\
- & \\
& \\
0,24 & (42) \\
0,33 & (42) \\
& \\
0,19 & (41)\end{array}$ & $\begin{aligned} 8,45 & (53) \\
23,64 & (52) \\
- & \\
0,45 & (71) \\
0,41 & (71) \\
0,30 & (70)\end{aligned}$ & $\begin{array}{r}1,98 \\
1,06 \\
- \\
\\
1,02 \\
1,14 \\
1,59\end{array}$ & $* *$ & $\begin{array}{l}0,160 \\
0,994 \\
0,361\end{array}$ & $*$ \\
\hline
\end{tabular}

- Différence significative à $90 \%$

** Différence significative à $95 \%$ 
Tab. 2. (suite) Résultats : moyenne des firmes utilisatrices et des non-utilisatrices Industries des produits de plastique, des ateliers d'usinage et de scierie

\begin{tabular}{|c|c|c|c|c|c|c|c|}
\hline & $\begin{array}{l}\text { Utilisatrices } \\
\text { du } \mathrm{CN}\end{array}$ & $\begin{array}{c}\text { Non-utilisatrices } \\
\text { du CN }\end{array}$ & $\begin{array}{l}\text { Ensemble } \\
\text { de } \\
\text { l'échantillon }\end{array}$ & $F$ & & $\begin{array}{l}\text { Signifi } \\
\text { du t }\end{array}$ & ativité \\
\hline $\begin{array}{l}\text { d) réalisation de rapports écrits pour } \\
\text { études de marché (oui ou non) } \\
\text { e) système de prix de revient } \\
\text { 3. Caractéristiques organisationnelles } \\
\text { - coordination et communication } \\
\text { a) présence d'un comité de gestion } \\
\text { b) tréquence des réunions (par mois) } \\
\text { - complexité } \\
\text { a) taille (emplois) }\end{array}$ & $\begin{array}{rr}0,72 & (29) \\
3,65 & (20) \\
80,90 & (29)\end{array}$ & $\begin{array}{rr}0,43 & (42) \\
1,94 & (18) \\
& \\
24,88 & (42)\end{array}$ & $\begin{array}{rr}0,55 & (71) \\
2,84 & (38) \\
& \\
47,76 & (71)\end{array}$ & $\begin{array}{r}1,21 \\
29,66 \\
\\
21,20\end{array}$ & ** & $\begin{array}{l}0,013 \\
0,250 \\
0,018\end{array}$ & ** \\
\hline
\end{tabular}

- Différence significative à $90 \%$

*" Différence significative à $95 \%$ 
Par contre, du côté des pratiques de gestion elles réalisent des études de marché souvent formalisées, elles recourent plus souvent à un comité de gestion et celui-ci se réunit beaucoup plus souvent. Elles utilisent donc de manière plus intensive des pratiques complexes de gestion.

On peut réaliser finalement que ces résultats respectent la plupart du temps les enseignements de la théorie discutés plus haut. Mais avant de poursuivre dans l'analyse de ces variables, nous nous attarderons un peu plus sur les sources d'informations, les objectifs des firmes et les barrières à l'innovation. Ces résultats sont aussi donnés en annexe.

\subsubsection{Les sources d'informations technologiques}

Comme cette variable semblait un élément-clé pour la diffusion des technologies, nous avons interrogé les entreprises visitées sur les sources d'informations qu'elles privilégiaientau point de vue technologique. Toutes entreprises confondues, le personnel de la firme, les foires industrielles, les revues spécialisées et leurs services de recherches, lorsqu'ils existent, se sont avérés les sources d'informations les plus fréquemment utilisées (annexe 2,p. 219). D'un autre côté, les médias traditionnels, les consultants, les organismes de recherche et les associations étaient les sources auxquelles elles avaient le moins souvent recours.

Les entreprises équipées du $\mathrm{CN}$ se sont distinguées quant à elles par leur recours significativement plus élevé aux foires industrielles et revues spécialisées, mais aussi aux fournisseurs ou aux fabricants, au personnel de vente, aux organismes des recherches et aux associations. De leur côté, les firmes sans $\mathrm{CN}$ se tournaient relativement plus fréquemment vers leur personnel, les revues spécifiques et les foires industrielles pour obtenir leurs informations technologiques. Ces dernières semblaient en comparaison nettement plus «introverties» en ce qui concerne la recherche d'informations.

\subsubsection{Les variables stratégiques}

Au niveau des variables stratégiques, nous avons observé que les entreprises que nous avons visitées s'appuyaient surtout sur la qualité du produit, son prix, le délai de livraison ainsi que la production de produits sur mesure pour assurer leur vente (annexe 3, p. 220). Au contraire la promotion ou les efforts de vente, la fabrication de produits dont l'abandon par les clients leur occasionnerait des coûts élevés de conversion et les facilités de crédit ont été plutôt considérés comme moins importants sinon mineurs.

Les entreprises qui utilisent la technologie de $\mathrm{CN}$, parmi ces variables ont insisté beaucoup plus sur les délais de livraison et sur l'image de firme fiable. Au contraire les entreprises non-utilisatrices misent en plus sur les facilités de crédit. 


\subsubsection{Objectifs des entreprises}

Au point de vue des objectifs futurs des entreprises, beaucoup ont à peu près la même importance, soit la recherche de nouveaux clients, la diminution des coûts, lacréation de nouveaux produits ou leur amélioration, l'expansion ou la consolidation des activités, et l'utilisation de nouvelles technologies (annexe 4, p. 221). Les entreprises utilisant des technologies à $\mathrm{CN}$ ont mis l'accent sur la diminution des coûts alors que les autres entreprises recherchaient plutôt des nouveaux clients.

Les objectifs les moins recherchés ont été le retrait de certaines productions, la fusion avec une autre entreprise ou l'acquisition d'un concurrent. Là aussi, la distinction n'est pas très grande entre les firmes plus modernes et les autres, même si l'objectif du retrait de certaines productions est discriminant.

\subsubsection{Les barrières à l'innovation}

Nous avons aussi obtenu des informations sur certains éléments que lesPME de notre échantillon considéraient comme des barrières ou des contraintes à l'introduction d'innovations technologiques ou de nouvelles technologies (annexe 5, p. 222).

La rentabilité insuffisante de la technologie en question, les risques financiers trop grands, les ressources financières déficientes et les problèmes de conversions coûteuses se sont avérés les principaux obstacles. Le potentiel commercial limité de l'innovation et les risques trop grands du point de vue marketing et de production sont aussi vus comme relativement importants. Il ressort aussi que les PME avec CN accordent une importance significativement moindre aux ressources financières insuffisantes. En moyenne d'ailleurs, les obstacles sont un peu moins importants que dans le cas des firmes sans $\mathrm{CN}$.

Les obstacles les moins importants étaient la répugnance à l'aide extérieure, une mauvaise communication dans l'entreprise, l'absence de procédures pour l'adoption ou l'implantation des nouvelles technologies et le manque d'idées nouvelles. La contrainte d'une mauvaise communication était significativement plus grande dans les $\mathrm{PME}$ avec $\mathrm{CN}$ que sans $\mathrm{CN}$.

\subsection{Résultats généraux}

Maintenant que nous avons pu identifier les variables qui semblaient différencier les PME utilisant le CN de celles qui ne l'avaient pas encore adopté, nous pouvons tracer un portrait de la PME à technologie de pointe dans les trois industries.

\subsubsection{Le profil des PME à technologie de pointe}

La firme à technologie de pointe est celle dont le propriétaire-dirigeant est nettement plus scolarisé. Il possède le plus souvent un baccalauréat et a une formation technique. En d'autres termes, les PME qui utilisent une innovation technologique 
(ici le $\mathrm{CN}$ ) sont celles dont le personnel avait une formation supérieure. Leurs cadres semblent également plus scolarisés et sont souvent ingénieurs.

En ce qui concerne les caractéristiques générales, ces entreprises nous sont apparues, encore une fois, plus cosmopolites ou plus tournées vers l'extérieur, plus ouvertes sur leur environnement et mieux informées sur les technologies disponibles. Leurs clients et fournisseurs ne sont pas seulement québécois ou canadiens mais se retrouvent aussi aux États-Unis et ailleurs dans le monde. Les sources d'informations qu'elles privilégient sont variées et externes à la firme. Leurs pratiques de gestion sont plus complexes; elles insistent entre autres sur la réalisation d'études de marché et recourent plus souvent à leur comité de gestion.

Compte tenu de leur allure générale, l'organisation de ces PME nous semble aussi plus complexe. Elles sont en effet plus grandes et disposent d'un personnel plus spécialisé et plus compétent en matière technique.

Les PME qui ne font pas usage du CN ont de leur côté un personnel moins scolarisé qui se contente d'études secondaires et collégiales. Leurs marchés et sources d'approvisionnements se trouvent en majorité au Québec et en Ontario et elles ont plus fréquemment recours à des sources internes d'informations, particulièrement au personnel de la firme. Elles ne sont pas intéressées outre mesure à collaborer avec des organismes de recherche et se limitent à surtout embaucher des techniciens plutôt que des ingénieurs.

Ce sont des entreprises de plus petite taille et moins complexes. Ces organisations semblent aussi plus introvertieset leur perspective surl'environnement sont plus restreintes. Leur propriétaire-dirigeant pourrait de plus s'assimiler d'une certaine manière, et bien que l'on ne possède pas formellement de données à ce sujet, à l'entrepreneur-artisan de Smith (1967). Celui de la PME «innovatrice» serait alors plus «opportuniste».

De plus, cette dernière catégorie de firmes accorde une grande importance aux délais de livraison et à l'image de la firme vis-à-vis de ses clients. Finalement des ressources financières insuffisantes sont pour elles un obstacle beaucoup moins important à l'introduction de nouvelles technologies. D'ailleurs la plupart des contraintes sont considérées comme moins importantes comparées à ce qu'en pensent les entreprises sans $\mathrm{CN}$.

Globalement, nos résultats n'ont rien de très singulier. Comme nous l'avons dit, ils vont sensiblement, pour la majorité d'entre eux, dans le sens de la théorie et des conclusions des principales recherches qui sont à la base de notre analyse. Le profil de la PME utilisant le CNO ressemble d'ailleurs passablement, pour ne pas dire énormément, à l'innovateur au sens large tel que l'ont décrit Rogers et Shoemaker, (1971 et 1975).

\subsubsection{Les facteurs d'adoption de nouvelles technologies}

Dans cette étude, nous ne nous sommes pas contentés de seulement faire le profil des firmes qui utilisaient le $\mathrm{CN}$ mais nous avons également voulu préciser 
l'importance des facteurs pour expliquer son adoption. Pour cela nous avons procédé à quelques traitements statistiques supplémentaires à l'aide de la technique de l'analyse discriminante.

Cette technique nous a permis d'ordonner les facteurs, des plus importants aux moins importants, permettant de bien différencier les entreprises utilisant le $\mathrm{CN}$ de celles n'y recourant pas. Comme on peut le voir au tableau 3, (p. 210), le recours à des sources d'informations technologiques spécialisées telles les fournisseurs et les foires industrielles, la présence d'un propriétaire-dirigeant possédant au moins un baccalauréat, l'utilisation d'un ordinateur, une taille plus grande que la moyenne, la réalisation d'études de marché avec rapport écrit et la collaboration avec des organismes de recherche constituent les facteurs les plus importants permettant de distinguer les entreprises utilisant des équipements avec $\mathrm{CN}$ de celles ne les utilisant pas. Ces sept facteurs permettent d'expliquer 82,8 \% des différences.

A ces facteurs, on peut en ajouter trois autres, ayant une importance secondaire bien que significative, et qui permettent d'ajouter presqu'un point pour expliquer les différences. Ces facteurs sont l'ouverture vers l'extérieur (taux d'importation et d'exportation plus élevé) et la présence d'un comité de gestion.

Ces résultats nous suggèrent donc des hypothèses intéressantes sur le processus d'adoption et les facteurs qui y entrent en jeu.

D'abord l'information technologique serait ainsi un déterminant crucial de la diffusion d'une innovation technologique de production. N'importe quel type d'information ne fait pas nécessairement l'affaire pour autant. Celle-ci doit être spécialisée et reliée aux besoins des firmes.

Disposer d'informations technologiques ne suffirait pas cependant. Il serait aussi avantageux de pouvoir apprécier avec justesse, par le biais entre autres d'études de marché et d'évaluation auprès d'organismes spécialisés, la situation et les perspectives des marchés et de l'environnement de l'entreprise, et les possibilités de production de technologies. Cecireviendraitàévaluer de manière préciselaprofitabilité et le potentiel d'utilisation d'innovations ou des technologies disponibles.

Cependant, c'est le rôle du propriétaire-dirigeant qui nous semble vital. Si une entreprise dispose d'informations, c'est d'abord parce qu'elle est «située» de manière à être bien exposée aux informations ou qu'elle possède les moyens, outils ou contacts nécessaires pour les recueillir, en un mot que sa gestion ou sa stratégie est «complexe». Dans ce cas, cette situation est redevable à son propriétairedirigeant. Celui-ci a aussi comme obligation de posséder les connaissances générales et techniques suffisantes pour utiliser et traiter ces informations, de s'entourer d'un personnel compétent pour l'y aider et de recourir à un bon comité de gestion pour le conseiller ou pour compléter ses connaissances. 
Tab. 3. Résultats de l'analyse discriminante

Industries des produits de plastique, des ateliers d'usinage et des scieries

1. V141 (source d'information : fournisseurs)

2. Ordin (utilisation d'un ordinateur)

3. V147 (source d'information : foires industrielles)

4. PDBAC (présence d'un propriétaire-dirigeant possédant un bacc.)

5. V355 (nombre d'emplois en 1986)

6. RAPPOR (réalisation d'études de marché avec rapport écrit)

7. ORGREC (collaboration avec des organismes de recherche)

\begin{tabular}{|llr|}
\hline R2 & $:$ & 0,5310 \\
Valeur d'Eigen & $:$ & 1,1355 \\
Corrélation canonique & $:$ & 0,7292 \\
Lambda de Wilks & $:$ & 0,4683 \\
\% des cas classés avec exactitude & $:$ & $82,76 \%$ \\
\hline
\end{tabular}

8. IMPOR1 (taux d'importation hors Québec)

9. EXPOR2 (taux d'exportation hors Québec et Ontario)

10. Comité (présence d'un comité de gestion)

\begin{tabular}{|llr|}
\hline R2 & $:$ & 0,5457 \\
Valeur d'Eigen & $:$ & 1,2044 \\
Corrélation canonique & $:$ & 0,7392 \\
Lambda de Wilks & $:$ & 0,4536 \\
\% des cas classés avec exactitude & $:$ & $83,33 \%$ \\
\hline
\end{tabular}

Par ailleurs, ce propriétaire-dirigeant està la tête d'une entreprise qui insiste beaucoup sur l'image de qualité et de fiabilité de l'entreprise et particulièrement sur la fabrication de produits typés ou sur mesure. L'utilisation du CN serait le reflet d'une volonté de maintenir ou d'atteindre le niveau «qualité» qu'exige la réalisation de ces produits comparativementà la production de masse. Dans une certaine mesure, ce point de vue confirmerait les idées généralement acceptées sur les apports stratégiques ou sur les impacts des nouvelles technologies sur la stratégie des entreprises.

Ce raisonnement sur le rôle du propriétaire-dirigeant et de l'information s'apparente de très près à certaines impressions qui se dégageaient lors de la visite d'entreprises n'utilisant pas le $\mathrm{CN}$. En prenant l'exemple d'une presse-à-injection, d'une fraiseuse ou d'un équarisseur, les propriétaires-dirigeants des entreprises plus traditionnellesplaçaient souvent la machine traditionnelle et celle à contrôle numérique sur un pied d'égalité, sauf pour ce qui touche le prix (le CNétanten moyenne au moins $50 \%$ plus coûteux). Conséquemment, en attribuant une valeur équivalente au $\mathrm{CN}$ 
mais à un prix plus élevé, ils en venaient toujours à conserver leurs équipements traditionnels. Une telle attitude révèle à notre avis une nette méconnaissance des technologies nouvelles. Il semble en effet que ces propriétaires-dirigeants ne soient guère conscients de tous les avantages de ces technologies : économies non seulement de main-d'oeuvre maisaussi de matièrespremières et de temps à cause dechangements plus rapides des outils, diminution des rejets ou augmentation de la qualité ou encore obtention d'une qualité plus uniforme, etc...

Dans le même ordre d'idée, certains résultats révèlent qu'un des principaux obstacles à l'innovation serait le risque financier ou les ressources insuffisantes, sans que des calculs de rentabilité qui tiendraient compte de tous les coûts et de tous les gains aient été effectivement effectués. Ceci tendrait à appuyer encore un peu plus la thèse expliquant l'absence du contrôle numérique par des déficiences au niveau de l'évaluation réelle de ces équipements ainsi qu'au niveau des informations sur leurs caractéristiques et leurs avantages.

Ajoutons enfin que ces résultats s'apparentent sur plusieurs aspects à d'autres résultats trouvés en France et en Belgique par De Bandt (1982), Wttervulghe (1986), l'équipe d'Alain d'Iribarne (1987) ou l'équipe de recherche Economie des changements technologiques de Lyon (Silem et alii, 1987).

Nous nous permettons de répéter que ces résultats ne traitent que du comportement de l'entreprise dans la diffusion des nouvelles technologies. Comme nous l'avons rappelé au début, l'environnement économique joue aussi un rôle certain. Nous croyons cependant que cette étude, malgré ses limites, peut être utile pour l'État dans ses programmes s'adressant aux firmes même si ces programmes ne sont pas suffisants.

\section{BIBLIOGRAPHIE}

Arthur D. Little Inc. et Industrial Research Institute Inc. (1973), Barriers to innovation in industry; opportunities for public policy changes, Wash. D.C., National Research Foundation.

Bouchut, Y. et Jacot, J.H., (1986), «Nouvelles technologies, crise et mutation du mode de production capitaliste», dans : Bouchut, Y. et alii, Nouvelles technologies et enjeux sociaux, PUL, Lyon.

Carter, C.F. et Williams, B.R., (1957), Industry and Technical Progress, London, Oxford University Press.

Carter, C.F. et Williams, B.R., (1959), «The characteristics of technically progressive firms», Journal of Industrial Economics, vol. 17, $\mathrm{n}^{\circ} 2, \mathrm{pp} .87-104$.

Cohn, S.F., (1979), «Characteristics of technically progressive firms», Omega vol. 8, n 4, pp. 441-450.

Cohn, S.F. et Turyn, R.M., (1980), «The structure of the firm and the adoption of progress innovations», IEEE Transactions on Engineering Management, EM-27, $\mathrm{n}^{\circ} 4$, novembre, pp. 98-102. 
Cohn, S.F.et Turyn, R.M., (1984), «Organizational structure, decision making procedures and the adoption of innovations», IEEE Transactions on Engineering Management, EM-31, $\mathrm{n}^{\circ} 4$, novembre, pp. 154-161.

Davies, W.S., (1979), The diffusion of process innovations, Londres, Cambridge University Press.

De Bandt, J., (1982), «Procédés nouveaux : une affaire de culture technique», Revue française de gestion, juin-juillet-août, pp. 51-56.

Duchesneau, T.D., Cohn, S.F. et Dutton, J.E., (1979), Diffusion of innovation; a longitudinal study, Wash. D.C., National Science Foundation.

Fisher, L.V., (1973), The diffusion of technological innovation, Londres, Polytechnic of Central London, vol. I et II

Gasse, Y., (1986), «Le processus d'adoption des nouvelles technologies par les PME», dans Julien, P.A., Joyal, A. et Chicha, J., (éds)., La PME en mutation, Québec, PUQ.

Gold, B., (1982), «Robotics, programmable programmation and international competitiveness», IEEE Transactions on Engineering Management, vol. 29, $\mathrm{n}^{\circ} 4$, novembre, pp. 135-146.

Gold, B., Peirce, W.S. et Rosseger, G., (1970), «Diffusion of major technological innovation in the U.S. iron and steel manufacturing», Journal of Industrial Economics, vol. 18, juillet, pp. 218-241.

Gruber, W.H. et Marquis, D.G., (éds), (1969), Factors in the transfer of technology, Cambridge, MA, The M.I.T. Press.

Hage, J. et Aiken, M., (1970), Social change in complex organisations, New York, Random House.

Hamberg, D, (1963), «Invention in the industrial research laboratory», Journal of Political Economy, vol. 71, avril, pp. 95-115.

Hayward, G., Allen, D.H. et Masterson, J., (1976), «Characteristics and diffusion of technological innovations,$: R$ \& $D$ Management vol. $7, \mathrm{n}^{\circ} 1$, octobre, pp. 15-24.

d'Iribarne, A., (1987), «Renouvellement technologique, PME et création d'emploi», dans «Diffusion des nouvelles technologies», numéro spécial de Interventions économiques.

Julien, P.A. et Hébert, L., (1986), «Le rythme de pénétration des nouvelles technologies dans les PME manufacturières», Journal of Small Business and Entrepreneurship, vol. 3, n ${ }^{\circ}$, pp. 24-35.

Kaplinsky, R., (1983), «Firm size and technical change in a dynamic context», The Journal of Industrial Economics, vol. 32, $\mathrm{n}^{\circ}$ 2, septembre, pp. 39-59.

Kennedy, C. et Thirwall, A.P., (1972), «Technical progress : a survey», Economic Journal, vol. 82, mars pp. 11-72.

Loinger, G., (1985) «La diffusion des innovations technologiques», Commissariat général au plan, Études et recherches, $\mathrm{n}^{\circ} 7$, Paris, La Documentation Française, juillet .

Malecki, E.J., (1975), Innovation diffusion among firms, Columbus, Ohio State University.

Mansfield, E., (1961), «Technical change and the rate of imitation», Econometrica, vol. 29, pp. 741-766. 
Mansfield, E., (1968), Industrial research and technological innovation, N.Y., W.W. Norton and Co.

Mansfield, E., (1971), Technological change, N.Y., W.W. Norton and Co.

Myers, S. et Marquis, D.G., (1969), Successfull industrial innovation, Wash. D.C., Institute of public administration

Nabseth, L., (1973), «The diffusion of innovation in Swedish industry», dans Williams, B.R. (ed.) Science and Technology in Economy Growth, Londres, McMillan Press.

OCDE, (1982), L'innovation dans les petites et moyennes entreprises, Paris, OCDE.

Olsen, R.P., (1974), Sources of process innovations in the textile industry, Cambridge, MA, Harvard Business School.

Pastré, O., Meyer, D., Trudel, J.L. et Zarader, R., (1981), «Informatisation, travail et emploi», dans Missika, J.L. et alii : Informatisation et emploi. Menace ou mutation ?, Paris, La Documentation Française.

Rogers, E.M. et Schoemaker, F.E., (1971), Communication of innovations, New York, The Free Press.

Rogers, E.M. et Eveland, J.D., (1975), «Diffusion : communication and innovation», dans Kelly et alii (eds), Technological innovation; a critical review of current knowledge, Georgia Tech : Advanced technology and science study group.

Rosenberg, N., (1974), «Science, invention and economic growth», Economic Journal, vol. 84, mars, pp. 90-108.

Rothwell, R., (1977), «The characteristics of successful innovatros and technically progressive firms», $R$ and $D$ Management, vol. 7, pp. 191-206.

Rothwell, R., (1984), «The role of small firms in the emergence of new technologies», Omega, vol. $12, \mathrm{n}^{\circ} 1$, pp. $19-29$.

Rothwell, R. et Zegveld, W., (1982), Innovations and the Small and Medium-Sized Firms, Hingham, MA, Gluwer-Nijhoff Publishing.

Smith, N.R., (1967), The entrepreneur and his firm; the relationship between type of man and type of company, East Landing, Michigan, Michigan State University.

Wiches, J.B., (1979), L'innovation technologique et la petite entreprise, Ottawa, MIC.

Wilson, J.O., (1966), «Innovation in organisation : notes toward a theory», dans Thompson, J.D. (éd.), Approaches to organizational design, Pittsburgh, PA, University of Pittsburgh Press.

Wttervulghe, R., (1986), «Thenew technologies and the small and medium-sized entreprises», SME : Keystone for a Free and Prosperous Europe, Tilburg, Tilburg University Press. 
Annexe 1

Variables et définitions opérationnelles

\begin{tabular}{|c|c|c|c|c|}
\hline Variables & $\begin{array}{l}\text { Relation entre la } \\
\text { variable et l'uti- } \\
\text { lisation du con- } \\
\text { tróle numérique }\end{array}$ & $\begin{array}{l}\text { Définitions } \\
\text { opérationnelles }\end{array}$ & $\begin{array}{l}\text { Relation entre la } \\
\text { définition opéra- } \\
\text { tionnelle et l'uti- } \\
\text { lisation du con- } \\
\text { trôle numérique }\end{array}$ & Commentaires \\
\hline $\begin{array}{l}\text { A) Caractérisques du } \\
\text { propriétaire-dirigeant } \\
\text { (Hypothèse 1) } \\
\text { - âge } \\
\text { - scolarité }\end{array}$ & 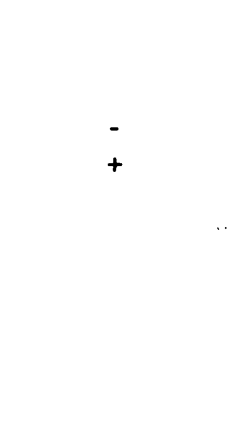 & $\begin{array}{l}\text { âge (années) } \\
\text { a) niveau d'étude le plus } \\
\text { élevé atteint (secondaire } \\
\text { (1), collégial (2), certificat } \\
\text { de premier cycle (3), } \\
\text { bacc. (4), maîtrise (5), } \\
\text { doctorat (6)) } \\
\text { b) détention d'un baccalauréat } \\
\text { (oui ou non) }\end{array}$ & $\begin{array}{l}- \\
+\end{array}$ & \\
\hline $\begin{array}{l}\text { - Formation techni- } \\
\text { que }\end{array}$ & + & $\begin{array}{l}\text { détention d'un diplóme technique } \\
\text { ou en génie (oui ou non) }\end{array}$ & + & \\
\hline $\begin{array}{l}\text { Attitude positive } \\
\text { vis-à-vis les chan- } \\
\text { gements technolo- } \\
\text { giques }\end{array}$ & + & $\begin{array}{l}\text { échelle de Nabseth (1973) } \\
\text { (1 à 5) }\end{array}$ & - & $\begin{array}{l}\text { Cinq énoncés } \\
\text { caractérisant cinq } \\
\text { comportements en } \\
\text { matière d'innovation }\end{array}$ \\
\hline
\end{tabular}


Annexe 1 (suite)

Variables et définitions opérationnelles

\begin{tabular}{|c|c|c|c|c|}
\hline Variables & $\begin{array}{l}\text { Relation entre la } \\
\text { variable et l'uti- } \\
\text { lisation du con- } \\
\text { trôle numérique }\end{array}$ & $\begin{array}{l}\text { Définitions } \\
\text { opérationnelles }\end{array}$ & $\begin{array}{l}\text { Relation entre la } \\
\text { définition opéra- } \\
\text { tionnelle et l'uti- } \\
\text { lisation du con- } \\
\text { trôle numérique }\end{array}$ & Commentaires \\
\hline $\begin{array}{l}\text { B) Caractéristiques des } \\
\text { cadres } \\
\text { (Hypothèse 2) } \\
-\quad \text { âge } \\
-\quad \text { scolarité }\end{array}$ & $\dot{-}$ & $\begin{array}{l}\text { âge moyen des cadres } \\
\text { a) moyenne du niveau d'étude le } \\
\text { plus élevé atteint (secondaire } \\
(1) \text {, collégial (2), certificat de } \\
\text { premier cycle (3), bacc. (4), } \\
\text { maîtrise (5), doctorat (6)) } \\
\text { b) \% des cadres ayant un bacca- } \\
\text { lauréat }\end{array}$ & + & \\
\hline \multirow[t]{3}{*}{$\begin{array}{l}\text { C) Caractéristiques géné- } \\
\text { rales et structurelles } \\
\text { des firmes } \\
\text { - disponibilité d'infor- } \\
\text { mations } \\
\text { (Hypothèse 3) }\end{array}$} & + & $\begin{array}{l}\text { a) taux d'exportation (\% des } \\
\text { ventes de } 1986 \text { réalisées à } \\
\text { l'extérieur du Québec) }\end{array}$ & + & $\begin{array}{l}\text { mesure utilisée par } \\
\text { Nabseth (1973) et } \\
\text { Nabseth et Ray (1974) }\end{array}$ \\
\hline & & $\begin{array}{l}\text { b) taux d'importation (\% des } \\
\text { achats de } 1986 \text { réalisés à } \\
\text { l'extérieur du Québec) }\end{array}$ & + & $\begin{array}{l}\text { application du même } \\
\text { raisonnement que pour } \\
\text { le taux d'exportation }\end{array}$ \\
\hline & & $\begin{array}{l}\text { c) nombre de fournisseurs de } \\
\text { la firme }\end{array}$ & + & $\begin{array}{l}\text { mesure utilisée par } \\
\text { Olsen (1974) }\end{array}$ \\
\hline
\end{tabular}


Annexe 1 (suite)

Variables et définitions opérationnelles

\begin{tabular}{|c|c|c|c|c|c|}
\hline & Variables & $\begin{array}{l}\text { Relation entre la } \\
\text { variable et l'uti- } \\
\text { lisation du con- } \\
\text { trôle numérique }\end{array}$ & $\begin{array}{c}\text { Définitions } \\
\text { opérationnelles }\end{array}$ & $\begin{array}{l}\text { Relation entre la } \\
\text { définition opéra- } \\
\text { tionnelle et l'uti- } \\
\text { lisation du con- } \\
\text { trôle numérique }\end{array}$ & Commentaires \\
\hline & & & $\begin{array}{l}\text { d) collaboration avec des orga- } \\
\text { nismes de recherche } \\
\text { (oui ou non) }\end{array}$ & + & \\
\hline & & & $\begin{array}{l}\text { e) fréquence d'utilisation de } \\
\text { sources privilégiées d'infor- } \\
\text { mation (littérature technique, } \\
\text { foire industrielle et four- } \\
\text { nisseurs) }\end{array}$ & + & $\begin{array}{l}\text { mesure inspirée de } \\
\text { Webster (1970); } \\
\text { échelle en cinq } \\
\text { points, de très rare- } \\
\text { ment à très souvent }\end{array}$ \\
\hline \multirow[t]{3}{*}{ - } & $\begin{array}{l}\text { Compétence techni- } \\
\text { que du personnel } \\
\text { (Hypothèse 4) }\end{array}$ & + & a) nombre d'ingénieurs & + & $\begin{array}{l}\text { mesure utilisée par } \\
\text { Cohn (1980) }\end{array}$ \\
\hline & & & b) nombre de techniciens & + & \\
\hline & & & c) $\%$ des cadres en production & + & $\begin{array}{l}\text { mesure utilisée par } \\
\text { Cohn (1980a) }\end{array}$ \\
\hline \multirow[t]{2}{*}{-} & $\begin{array}{l}\text { Croissance de la } \\
\text { firme } \\
\text { (Hypothèse 5) }\end{array}$ & + & $\begin{array}{l}\text { a) taux de croissance annuel } \\
\text { moyen des ventes entre } \\
1981 \text { à } 1986\end{array}$ & + & \\
\hline & & & $\begin{array}{l}\text { b) taux de croissance annuel } \\
\text { moyen de l'emploi entre } \\
1981 \text { et } 1986\end{array}$ & + & \\
\hline
\end{tabular}


Annexe 1 (suite)

Variables et définitions opérationnelles

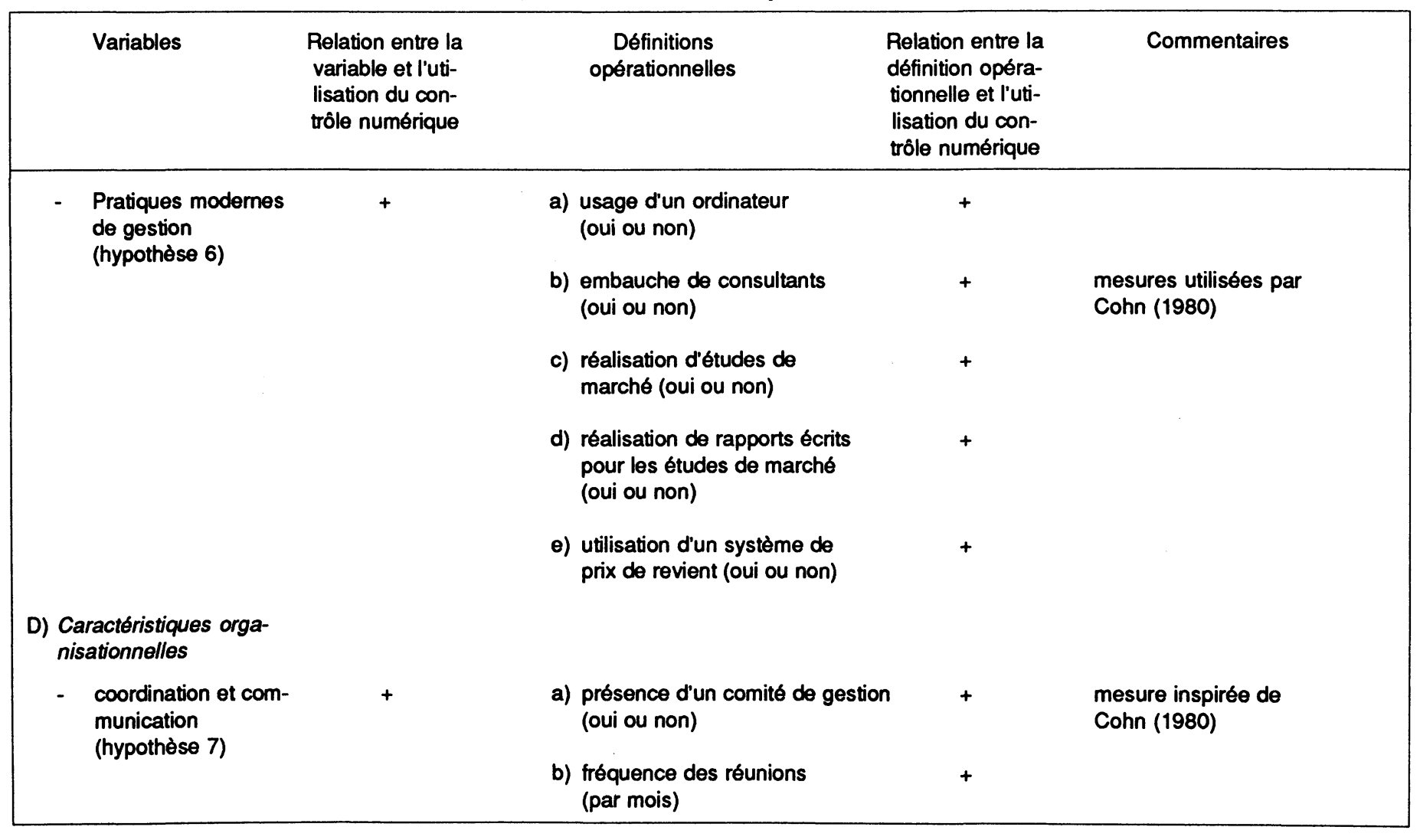


Annexe 1 (suite)

Variables et définitions opérationnelles

\begin{tabular}{|c|c|c|c|c|c|}
\hline & Variables & $\begin{array}{l}\text { Relation entre la } \\
\text { variable et l'uti- } \\
\text { lisation du con- } \\
\text { trôle numérique }\end{array}$ & $\begin{array}{c}\text { Définitions } \\
\text { opérationnelles }\end{array}$ & $\begin{array}{l}\text { Relation entre la } \\
\text { définition opéra- } \\
\text { tionnelle et l'uti- } \\
\text { lisation du con- } \\
\text { trôle numérique }\end{array}$ & Commentaires \\
\hline \multirow[t]{2}{*}{ - } & $\begin{array}{l}\text { complexité } \\
\text { organisationnelle } \\
\text { (hypothèse 8) }\end{array}$ & + & a) taille (nombre d'emplois) & + & $\begin{array}{l}\text { mesure suggérée par } \\
\text { Duchesneau et alii } \\
\text { (1979) }\end{array}$ \\
\hline & & & $\begin{array}{l}\text { b) échelle du niveau d'activité } \\
\text { professionel des cadres à } \\
\text { l'extérieur de la firme (sur 4) }\end{array}$ & + & $\begin{array}{l}\text { échelle à quatre énon- } \\
\text { cés utilisée par Hage } \\
\text { et Aiken (1967) }\end{array}$ \\
\hline
\end{tabular}


Annexe 2

Fréquence d'utilisation des sources d'information technologique

Industries des produits de plastique, des ateliers d'usinage et des scieries

\begin{tabular}{|c|c|c|c|c|c|c|c|c|}
\hline \multirow[b]{2}{*}{ Recherche et Développement } & \multirow{2}{*}{$\begin{array}{c}\begin{array}{c}\text { Ensemble } \\
\text { de } \\
\text { l'échantillon }\end{array} \\
2,74(70)\end{array}$} & \multicolumn{2}{|c|}{$\begin{array}{c}\text { PME } \\
\text { Utilisatrices } \\
\text { du CN }\end{array}$} & $\begin{array}{c}\text { PME } \\
\text { Non-utilisatrices } \\
\text { du CN }\end{array}$ & \multicolumn{2}{|c|}{$F^{2}$} & \multicolumn{2}{|c|}{ Probabilité $^{3}$} \\
\hline & & 2,79 & (29) & $2,71(41)$ & 1,07 & & 0,815 & \\
\hline Personnel de la firme & $3,51 \quad(70)$ & 3,63 & (29) & $3,44(41)$ & 2,53 & ** & 0,418 & \\
\hline Personnel de vente & $2,43(70)$ & 2,83 & (29) & $2,15(41)$ & 1,05 & & 0,052 & * \\
\hline Fournisseurs & $2,44(70)$ & 3,10 & (29) & $1,98(41)$ & 1,13 & & 0,000 & \#* \\
\hline Concurrents & $2,06(70)$ & 2,28 & (29) & $1,90(41)$ & 1,54 & & 0,198 & \\
\hline Clients & $2,63(70)$ & 2,76 & (29) & $2,54(41)$ & 1,17 & & 0,412 & \\
\hline Autres entreprises & $2,66(70)$ & 2,93 & (29) & $2,46(41)$ & 1,26 & & 0,120 & \\
\hline Revues spécialisées & $2,93(70)$ & 3,03 & (29) & $2,86(41)$ & 1,00 & & 0,549 & \\
\hline Médias (autres) & $1,54(70)$ & 1,62 & (29) & $1,49(41)$ & 1,43 & & 0,472 & \\
\hline Foires industrielles & $3,01(70)$ & 3,45 & (29) & $2,71(41)$ & 1,23 & & 0,013 & $*$ \\
\hline Fabricants & $2,57(70)$ & 2,90 & (29) & $2,34(41)$ & 1,17 & & 0,046 & \#* \\
\hline Associations & $2,04(70)$ & 2,31 & (29) & $1,85(41)$ & 1,21 & & 0,070 & * \\
\hline Consultants & $1,90(70)$ & 2,03 & (29) & $1,80(41)$ & 1,19 & & 0,396 & \\
\hline Organismes de recherche & $2,01(70)$ & 2,48 & (29) & $1,68(41)$ & 1,65 & & 0,005 & ** \\
\hline Autres & $1,41 \quad(64)$ & 1,52 & $(25)$ & $1,33(39)$ & 1,35 & & 0,450 & \\
\hline
\end{tabular}

- Différence significative à $90 \%$

* Différence significative à $95 \%$

1. Le chiffre entre parenthèses nous donne le nombre de PME ayant répondu

2. Coefficient de Fisher

3. Valeur de $T$ 
Annexe 3

Variables stratégiques

Industries des produits de plastique, des ateliers d'usinage et des scieries

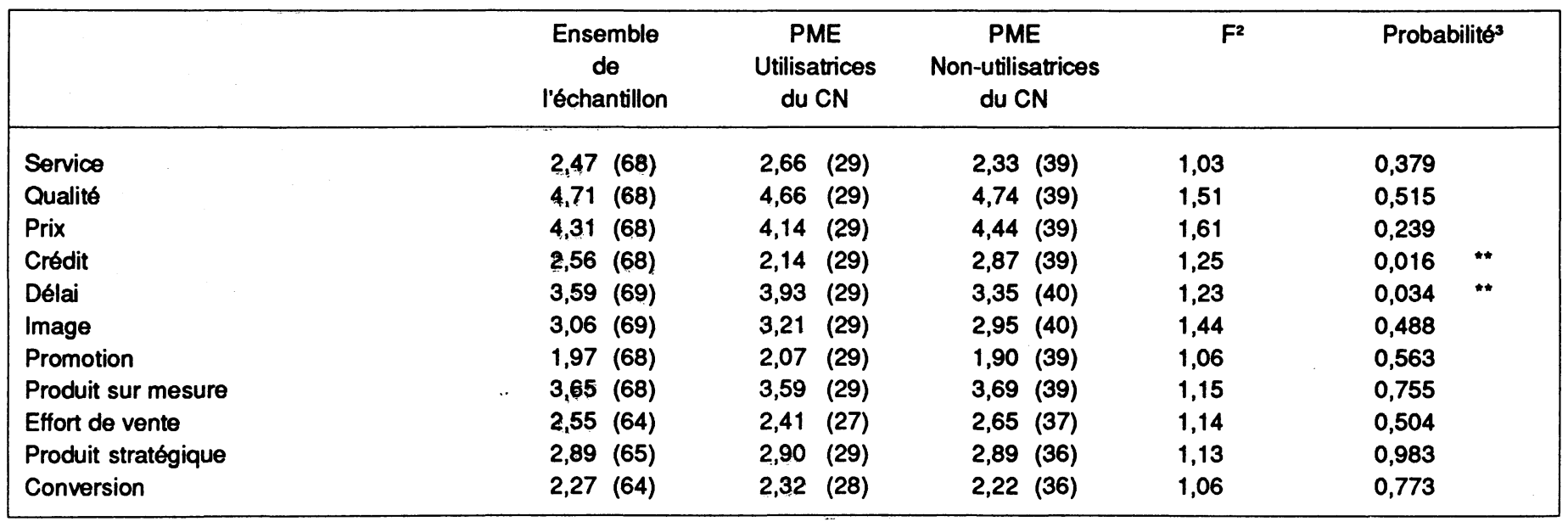

- Différence significative à $90 \%$

** Différence significative à $95 \%$

1. Le chiffre entre parenthèses nous donne le nombre de PME ayant répondu

2. Coefficient de Fisher

3. Valeur de $\mathrm{T}$ 
Annexe 4

Importance des objectlifs futurs

Industries des produits de plastique, des ateliers d'usinage et des scieries

\begin{tabular}{|c|c|c|c|c|c|c|c|c|}
\hline \multirow[b]{2}{*}{ Coûts } & \multirow{2}{*}{$\begin{array}{c}\begin{array}{c}\text { Ensemble } \\
\text { de } \\
\text { l'échantillon }\end{array} \\
3,40(70)\end{array}$} & \multicolumn{2}{|c|}{$\begin{array}{c}\text { PME } \\
\text { Utilisatrices } \\
\text { du } \mathrm{CN}\end{array}$} & $\begin{array}{c}\text { PME } \\
\text { Non-utilisatrices } \\
\text { du CN }\end{array}$ & \multicolumn{2}{|c|}{$F^{2}$} & \multicolumn{2}{|c|}{ Probabilité $^{3}$} \\
\hline & & 3,54 & (28) & $3,31 \quad(42)$ & 1,05 & & 0,508 & \\
\hline Consolidation & $3,23(70)$ & 3,14 & (28) & $3,29(42)$ & 1,11 & & 0,695 & \\
\hline Acquisition & $1,58(71)$ & 1,76 & (29) & $1,45(42)$ & 1,66 & & 0,236 & \\
\hline Fusion & $1,30(70)$ & 1,25 & (28) & $1,33(42)$ & 4,43 & ** & 0,617 & \\
\hline Expansion & $3,33(70)$ & 3,21 & (28) & $3,41 \quad(42)$ & 1,08 & & 0,613 & \\
\hline Nouveaux produits & $3,38 \quad(69)$ & 3,21 & (28) & $3,49 \quad(41)$ & 1,06 & & 0,474 & \\
\hline Amélioration produits & $3,28 \quad(69)$ & 3,21 & (28) & $3,32(41)$ & 1,05 & & 0,755 & \\
\hline Nouvelles technologies & $3,25(69)$ & 3,25 & (28) & $3,24(41)$ & 1,24 & & 0,985 & \\
\hline Nouveaux clients & $3,56(68)$ & 3,29 & (28) & $3,75(40)$ & 1,39 & & 0,102 & \\
\hline Diversification & $2,54(69)$ & 2,39 & (28) & $2,63(41)$ & 1,22 & & 0,454 & \\
\hline Retrait & $1,18 \quad(68)$ & 1,33 & $(27)$ & $1,07(41)$ & 1,36 & & 0,091 & $\star$ \\
\hline Pénétration & $2,91 \quad(69)$ & 2,86 & (28) & $2,95(41)$ & 1,22 & & 0,786 & \\
\hline
\end{tabular}

- Différence significative à $90 \%$

** Différence significative à $95 \%$

1. Le chiffre entre parenthèses nous donne le nombre de PME ayant répondu

2. Coefficient de Fisher

3. Valeur de $T$ 
Annexe 5

Barrières et contraintes à l'innovation

Industries des produits de plastique, des ateliers d'usinage et des scieries

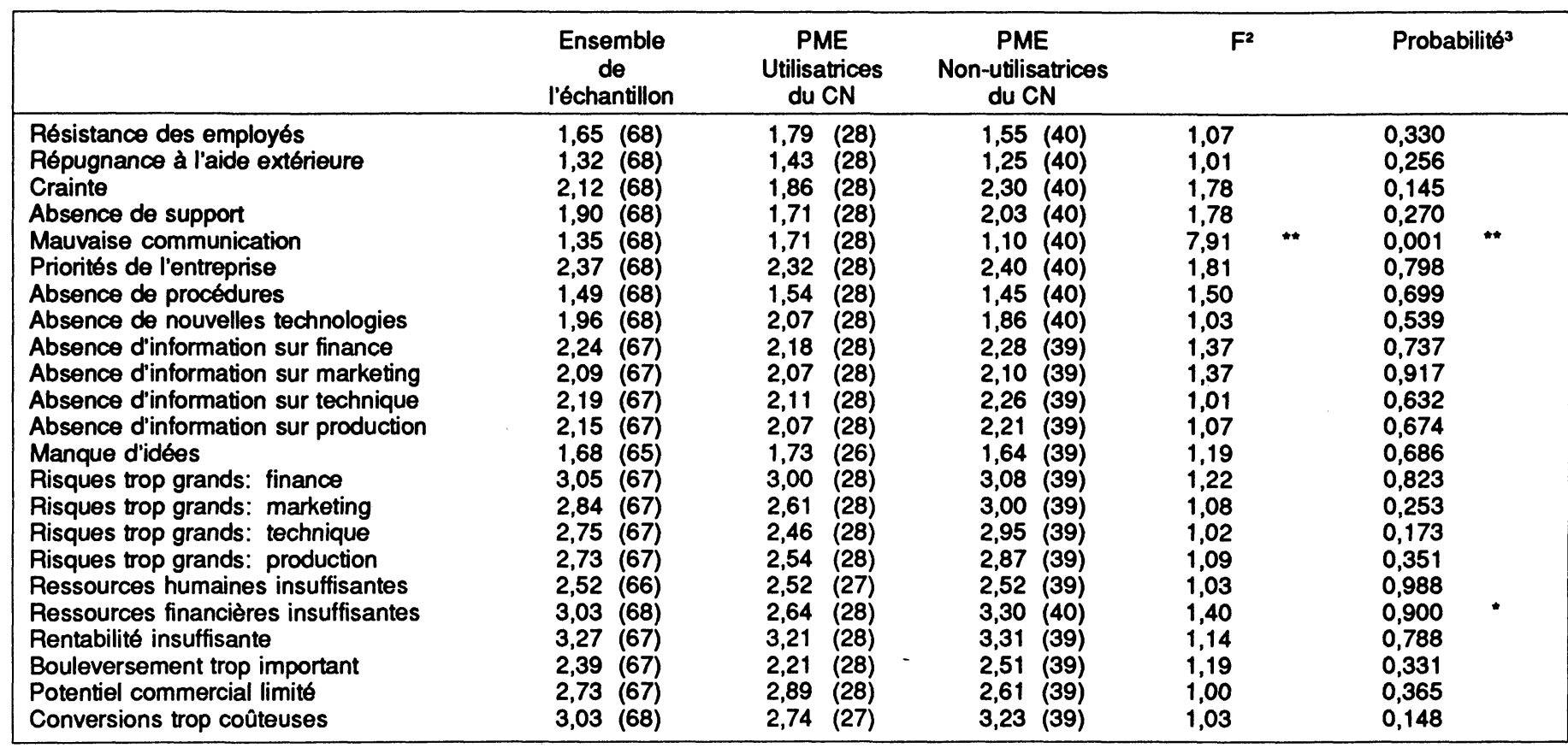

* Différence significative à $90 \%$ _ * Différence significative à $95 \%$

1. Le chiffre entre parenthèses nous donne le nombre de PME ayant répondu

2. Coefficient de Fisher

3. Valeur de $T$ 